\title{
Desafíos de la historia oral en las sociedades oprimidas: caso cubano
}

\section{Challenges of oral history in repressed societies: the Cuban case}

\author{
Izabela Tkocz* \\ Jesús Adolfo Trujillo Holguín**
}

* Profesora de la Universidad Autónoma de Chihuahua, México. Es doctora en Educación, Artes y Humanidades. Entre sus publicaciones recientes están "Movimientos estudiantiles en Polonia y México de 1968: un estudio comparativo" en Revista Izquierdas (2020) y “Qué valió la pena? 30 años de la Mesa Redonda en Polonia 19892019. La caída del bloque socialista en Europa del Este y la memoria histórica” en Revista Mexicana de las Ciencias Politicas y Sociales (2020). Pertenece al Sistema Nacional de Investigadores. Sus temas de interés son Historia e historiografía del siglo $X X$, historia critica, arte e historia, geopolitica, metodología de la investigación histórica. Correo electrónico: itkocz@uach.mx

https://orcid.org/0000-0002-3973-2888

* Profesor-investigador de la Universidad Autónoma de Chihuahua, México. Es doctor en educación y cuenta con especialidad en competencias docentes por la Universidad Autónoma de Madrid (España). Entre sus publicaciones recientes están los libros "La educación en Ranchería Juárez, Chihuahua, 1932-2018” (2019) y "Desarrollo profesional docente: reflexiones y experiencias de inclusión en el aula” (2020). Cuenta con los reconocimientos al perfil Prodep y del Sistema Nacional de Investigadores, Nivel 1. Es socio del Consejo Mexicano de Investigación Educativa, de la Red de Investigadores Educativos Chihuahua y presidente de la Sociedad Mexicana de Historia de la Educación (2019-2021). Correo electrónico: jatrujillo@uach.mx

http://orcid.org/0000-0002-6738-1878

Historial editorial

Recibido: 19-mayo-2020

Aceptado: 02- diciembre-2020

Publicado: 29-enero-2021

ISSN-e: 2594-2956 
Desafíos de la historia oral en las sociedades oprimidas: caso cubano

\section{Resumen}

El artículo hace referencia a los problemas relacionadas con la historia oral y su ejecución en las sociedades oprimidas, caso cubano. El trabajo se concentró en las entrevistas realizadas de manera casual y aleatoria, durante una estancia académica en la isla, realizada entre noviembre $y$ diciembre de 20I8. El objetivo principal de la investigación era encontrar y analizar la influencia de la propaganda soviética en la educación y cultura cubana, pero durante las indagatorias aparecieron adicionalmente las cuestiones relacionadas con la historia y la vida cotidiana después de la Revolución cubana. Al mismo tiempo, surgieron también los problemas con la verificación de los datos proporcionados, pues las fuentes oficiales, controladas totalmente por el Estado, omitían mucha información que de cierto modo podía ser incómoda para el gobierno. Por lo tanto, pareció pertinente reflexionar acerca de rol

156 y de la metodología de la historia oral en las sociedades oprimidas.

Palabras clave: historia oral, investigación, metodología, vida cotidiana, Cuba.
Challenges of oral history in repressed societies: the Cuban case

\section{Abstract}

This article focuses on the problems and potentials of oral history in repressed societies, the Cuban case. The work presented here is based on interviews carried out in a random and casual way during a stay on the island of Cuba from November to December 2018. The main objective of the research initially was to find and analyze the influences of Soviet propaganda on Cuban education and culture, but during the investigations, issues related to history and daily life after the Cuban revolution also appeared. Nevertheless, verification of the data provided by informants is problematic since official sources are totally controlled by the state and omit information that is uncomfortable for the government. Therefore, it is considered pertinent to reflect on the role and methodology of oral history in repressed societies

Keywords: oral history, research, methodology, daily life, Cuba. 
Défis de l'histoire orale dans les sociétés opprimées : cas cubain.

\section{Résumé}

L'article fait référence aux problèmes liés à l'histoire orale et à son exécution dans les sociétés opprimées, cas cubain. Le travail s'est concentré sur les entretiens effectués de manière aléatoire et aléatoire pendant le séjour sur l'île entre novembre/décembre 2018. L'objectif principal de l'enquête était de découvrir et d'analyser les influences de la propagande soviétique sur l'éducation et la culture cubaines, Mais au cours des enquêtes, les questions liées à l'histoire et à la vie quotidienne après la révolution cubaine sont apparues. Dans le même temps, des problèmes ont surgi avec la vérification des données fournies, car les sources officielles entièrement contrecarrées par l'État omettaient beaucoup d'informations qui pourrait en quelque sorte être inconfortable pour le gouvernement. Il a donc semblé pertinent de réfléchir au rôle et à la méthodologie de l'histoire orale dans les sociétés opprimées.

Mots-clés: histoire orale, recherche, méthodologie, vie quotidienne, Cuba.
Wyzwania historii ustnej w społeczeństwach kontrolowanych przez system polityczny: Kuba

\section{Streszczenie}

Artykuł odnosi się do problemów związanych $\mathrm{z}$ historią ustna $\mathrm{i}$ jej egzekucją w uciśnionych społeczeństwach, na przykladzie Kuby. Prace koncentrowały się na wywiadach przeprowadzonych podczas pobytu na wyspie w okresie od listopada do grudnia 2018 roku. Głównym celem badań było znalezienie wpływów sowieckiej propagandy na kubańską edukację i kulturę, i jej analiza. Jednakze podczas wywiadow pojawily sie nowe kwestie związane $\mathrm{z}$ historią $\mathrm{i}$ codziennym życiem po rewolucji kubańskiej. Jednocześnie pojawiły się problemy $\mathrm{z}$ weryfikacją dostarczonych danych, ponieważ oficjalne źródła całkowicie zdominowane przez państwo pomijały wiele informacji, które mogą być w jakiś sposób niewygodne dla rządu. W związku z tym kwestia roli $\mathrm{i}$ metodologii historii ustnej w uciśnionych społeczeństwach wydawała się istotna.

Slowa kluczowe: historia ustna, badania, metodologia, życie codzienne, Kuba. 


\section{Introducción}

En el presente artículo se abordan los desafíos de la historia oral en las sociedades oprimidas, enfocando el análisis en la problemática para "construir" el relato en el contexto cubano. Las actividades de investigación se realizaron en el periodo de noviembre a diciembre de 20I8, como parte de una estancia académica en la Universidad de La Habana, cuyo propósito fue la búsqueda de información referente a la influencia de la propaganda socialista soviética en la cultura y educación en Cuba.

De la colección de más de 40 entrevistas realizadas con personas de la Isla, solo 9 estaban relacionadas con el tema principal del trabajo y el resto abarcaron diferentes temas relacionados con la vida bajo el régimen postcastrista: ${ }^{\mathrm{I}}$ economía, política, sistema de salud, entre otros. En principio se temía que fuera difícil obtener información de fuentes orales, tomando en cuenta la experiencia personal de la autora principal de este trabajo, quien vivió en el régimen socialista del Bloque del Este, específicamente en Polonia. Sin embargo, en el caso cubano, la mayoría de las personas, a pesar de su aprensión inicial, posteriormente relataron sus historias de vida con destacable franqueza, incluso cuando su posicionamiento contradecía el discurso oficial del Estado.

Se cree que en los países socialistas la historia oral es una metodología casi fallida porque la gente teme hablar sobre su vida, como ocurrió en varios intentos que se hicieron en países del Bloque del Este, donde los investigadores solo lograron recuperar testimonios orales cuando los entrevistados vivían en el exilio. Como es de esperar, la historia oral fue escasa en países más allá del Muro de Berlín, pero floreció más tarde,

158 cuando la población empezó a demandar que sus historias fueran escuchadas (Bertaux, Rotkirch y Thompson, 2004).

Para el caso cubano la experiencia fue diferente. Las y los entrevistados hablaron sobre la vida en Cuba, así como de una cuestión polémica: la creciente desigualdad, que ha generado sentimientos encontrados en 
los ciudadanos y que da cuenta de las condiciones sociales de la Cuba actual.

Hay muy pocos trabajos que abordan el tema de la historia oral en Cuba. Uno de los más conocidos es el proyecto de Oscar Lewis acerca de la vida cotidiana, que inició en 1969 con la aprobación especial de Fidel Castro. Se suponía que Lewis y su equipo estarían en la Isla durante tres años, pero abruptamente el proyecto se redujo a un año y medio, cuando murió Oscar. Buena parte del material que habían reunido fue confiscado por el gobierno cubano y de lo que se logró rescatar, su esposa Ruth -quien le acompañaba en la investigaciónpreparó dos libros: Four men: Living the Revolution. An Oral History of Contemporary Cuba y Four women: Living the Revolution. An Oral History of Contemporary Cuba, que se publicaron en 1977 (Lewis, Lewis \& Rigdon, I977a y 1977 b).

El gobierno castrista se posicionó respecto a la presencia de los investigadores extranjeros en el país, como señala Lewis (1980):

En 1972, en un discurso sobre problemas de seguridad interna, Raúl Castro Ruz, Vice-primer Ministro del Ejército Revolucionario, y hermano menor de Fidel, se extendió sobre el tema general del "colonialismo cultural" y los extranjeros peligrosos. En este discurso, dos años después de que salimos de Cuba, Oscar, fue retratado, junto con otros más, como agente secreto: "Una parte de los visitantes de países capitalistas — sociólogos, profesores, periodistas, marinos y turistas - suelen ser agentes de información o colaboradores de los servicios enemigos" (p. I5).

Otro trabajo de historia oral fue de Gabriel García Márquez, quien inicia -en 1975- con la recuperación de los recuerdos acerca de la Revolución, pero "Un año después abandonó el proyecto porque, de acuerdo con lo que les comentó a sus amigos, lo que la gente decía no encajaba con el libro que tenía en mente" (Dore, 2012, A contracorriente, párr. 3).

Elizabeth Dore tenía otro proyecto de historia oral que también se haría con el permiso del gobierno cubano -autorizado en 2003- pero la 
investigación empezó hasta 2005. Se canceló cuando los informantes empezaron a contar historias diferentes a la versión oficial. En su investigación, Dore observó procesos semejantes a los que se viven actualmente, como son desigualdad social, apego al mito de Fidel y el rol de la entrevista como una especie de catarsis (Dore, 20I2).

En el caso polaco, el desarrollo de la historia oral se relaciona con las rebeliones contra el sistema, en los años de 1956 y 1968, cuando se dificultó publicar en los medios oficiales los relatos de los testigos que contradecían el discurso estatal. Un grupo de historiadores y periodistas comenzaron a escribir y grabar estas historias, esperando que en el futuro los tiempos favorecieran la libre expresión de distintos puntos de vista. La primera publicación oficial fue de la autoría de Krystyna Kersten -titulada Relatos como fuente histórica (I969)- donde menciona que las fuentes orales son un material adicional para el trabajo del historiador y deberían estar complementados con conocimientos de psicología y sociología (Dabrowski, 2008).

En I98I, después de las grandes huelgas que ocurrieron un año antes, el historiador Tadeusz Łepkowski empezó a realizar trabajos a gran escala, basados en la historia oral. Recuperó los testimonios, no solo de disidentes, sino de gente común y corriente, acerca de la vida en tiempos de Solidaridad. En un inicio, la investigación se limitaba a acontecimientos políticos y así se publicaron: Poznan, junio 1956 de Jarosław Maciejewski i Zofia Trojanowicz (I98I), Ellos de Teresa Torańska (1985) y diciembre 1970 a cargo de una organización no gubernamental fuera del país (I986) (Dabrowski, 2008).

Para el presente trabajo de investigación se pudo constatar que la historia oral en Cuba y Polonia tuvo los mismos problemas para su inicio y desarrollo, considerando las condiciones de un Estado autoritario que ejercía -y aun ejerce- control de la información y trata a los investigadores como enemigos potenciales. La diferencia principal reside en la apertura de los entrevistados cubanos para narrar sus historias de vida, pues rompieron con el esquema del Bloque del Este, donde las personas se reservaban para sí sus experiencias. 


\section{¿Qué es la Historia Oral?}

La historia oral es una de las ramas más jóvenes de la historia y a la vez de mayor complejidad. Su uso no se limita al campo de la historia, sino que también cuenta con adeptos en disciplinas como la antropología, etnografía, sociología, pedagogía, psicología, literatura, lingüística, entre muchas otras. Prácticamente cada uno de nosotros hemos tenido por lo menos un acercamiento a la historia oral dentro de la familia, al hablar con los abuelos, tíos, padres, etcétera; o en el ámbito público con políticos, actores o escritores. La tradición oral aparece desde tiempos muy remotos, incluso antes de la invención de la escritura.

La historia oral ha sido muy debatida y sus términos no están suficientemente bien establecidos. Uno de los desafíos es precisar los conceptos (Sebe, 1998a), pues sus bases teóricas fueron formuladas en Estados Unidos después de la Segunda Guerra Mundial, pero ya desde la década de 1920 los miembros de la Escuela de Sociología de Chicago empezaron a experimentar con ella, no como parte de la historia, sino como un método complementario que les permitía investigar las derivaciones sociales (Riemann y Schütze, 1992).

La primera institución que elaboró el un proyecto de historia oral fue la Columbia University, donde se creó el centro la Oral History Research Office en 1948. Su fundador fue Allan Nevins. Al principio los historiadores orales estaban convencidos que se debían registrar los recuerdos de las personas públicas importantes, pero en los años sesenta cambió la idea y los investigadores se concentraron en las memorias de las clases populares y los grupos olvidados, quienes no habían sido tomados en cuenta durante mucho tiempo (Dabrowski, 2008).

Entre los años I954 a I972 se creó el proyecto del Centro de Historia Oral en la Universidad de Misisipi, el cual concentró sus esfuerzos en la historia de los movimientos pro derechos humanos y veteranos de guerra. Sin embargo, uno de los cambios más significativos fue en 1968, con los movimientos estudiantiles que se intensificaron en todo el mundo y los cuales manifestaban desacuerdo con el orden político y 
social imperante hasta entonces. Una de las consecuencias de estos movimientos fue la fundación del Proyecto de Historia Oral en la Universidad de Connecticut, que trató de recuperar la memoria de los involucrados en las protestas y, posteriormente, concentró su interés en la historia local (Dabrowski, 2008).

En Europa, el primer estudio serio fue el de Jan Vansina, titulado Oral Tradition as History (196I). Con él se revalorizaron las entrevistas y se estableció el contacto entre la historia y otras ciencias sociales como la sociología, antropología y etnología (Thompson, 1978).

Los años sesenta revivieron las discusiones sobre la historia oral, especialmente cuando los historiadores con orientación de izquierda propusieron dar voz a los grupos no privilegiados y olvidados. Los pioneros de este proyecto fueron Paul Thompson, Trevor Lummis y Daniel Bertaux.

El siguiente giro de la historia oral se dio en los años ochenta, cuando Alessandro Portelli y Luisa Passerini comprobaron que las fuentes orales, que muchas veces no son verídicas, también podían ser material de investigación (Dabrowski, 2008). En I982 se estableció en Birmingham, Gran Bretaña, el llamado Popular Memory Group y los métodos que utilizaron fueron trasladaron hacia otras disciplinas científicas. También fue influyente en el decenio de 1970 el History Workshop de Raphael Samuel (Anderson \& Blackledge, 2004), los investigadores involucrados en la "historia desde abajo" y la revista Past $\mathcal{E}$ Present.

El tercer gran movimiento dentro de la historia oral fue la narratología, que se popularizó en Alemania. Sus representantes (Gabriele

162 Rosenthal, Fritz Schütze y Alexander von Plato) trataban la narración como una construcción que evolucionaba a la biografía (Riemann y Schütze, 1992). Desde los años de I980 destacó por su trabajo y metodología Lutz Niethammer.

Jerzy Topolski (1983), en su trabajo Teoría del conocimiento histórico, vio la historia oral de una manera más amplia, no solo como relatos 
obtenidos por el investigador, sino como la tradición oral y las memorias escritas por los historiadores.

En México los principales exponentes de la historia oral -por ejemplo, Jorge Aceves, Mario Camarena o Gerardo Necoechea- se concentraron también en captar las historias de los grupos olvidados y marginados: obreros, mujeres, guerrilleros, integrantes de pueblos indígenas o dentro de la historia de la educación (Sebe, 1998a). Desde fines de los años de 1960 a través del INAH hubo trabajos para recopilar los testimonios de los combatientes zapatistas en la Revolución mexicana.

Actualmente la historia oral se caracteriza por concentrar su interés en la manera en que se transmite el relato. Gracias a este cambio, comenzaron a utilizar y analizar el material obtenido en otras disciplinas científicas. Así se convirtió en un pretexto para estudiar la memoria individual y colectiva, y la manera en que se construye la narración histórica, los mitos o los estereotipos. La tendencia de utilizar la oralidad dentro de la sociología y la antropología es especialmente vista dentro de la Gran Bretaña. Este tipo de estudios se relacionaron con los movimientos de 1968. El método que se usó se acuñó como entrevista en profundidad (Dabrowski, 2008). También antes del decenio de 1930 existieron los Mass Culture Observation como bien ha relatado Dick Hebdige (1979), aunado al surgimiento de los Cultural Studies en la Universidad de Birhminghan, encabezados por Stuart Hall, que dieron lugar al Centro de Estudios Culturales Contemporáneos. Todo esto formó parte del contexto de institucionalización y promoción de la historia oral y otras técnicas para recopilar información por esa vía.

Una de las contribuciones fundamentales de la historia oral al debate académico actual es en relación al papel del documento, al reconocer que los testimonios orales enaltecen la importancia del historiador como creador de textos, que luego serán la base para otras investigaciones.

La historia oral se caracteriza por incluir dos tiempos: el primero de transformación de la palabra en documento escrito; el segundo, de 
reflexión sobre el contenido. El momento en que el historiador elabora un documento escrito significa asumir la responsabilidad con la sociedad. Ya no es un agente pasivo que utiliza la documentación elaborada; el juicio crítico de la fuente escrita se hace personal (Sebe, I998a).

El testimonio con el cual trabaja el investigador es más que una entrevista casual. La historia oral es algo más que una simple conversación grabada, pues implica el uso de gran cantidad de recursos metodológicos que exigen una calificación personal para tratar el tema. Abarca desde la construcción del proyecto hasta la elaboración del documento final, que a la vez es uno de los compromisos devolverlo hacia la comunidad que lo generó. No se puede confundir memoria con historia oral, pues la segunda se compromete con la comunidad que produce y vive su propia historia, y con la conversión del relato en documento, por eso se dice que es una rama de la historia pública (Sebe, I998a).

La historia oral se divide en tres ramas: Historia oral de vida, Historia oral temática y Tradición oral, todas se basan en los testimonios, pero a la vez se distinguen de las entrevistas periodísticas, sociológicas, antropológicas, etcétera. La Historia oral de vida remite al registro de la experiencia personal. Georges Gusdorf (1957) se interesó por las autobiografías, cartas y relatos personales escritos en primera persona; íntimamente ligados a los documentos del yo. Dentro de la metodología, se les conoce como entrevistas libres, es decir, sin preguntas dirigidas y sin cuestionario. Se distinguen porque captan la vivencia y experiencia de alguien y por ello suelen ser muy largas. Todos los fragmentos y detalles de la vida del informante tienen una importancia relativa.

La rama correspondiente a la Historia oral temática compite con los textos escritos porque se vincula al interés por un tema específico en el que se abordan cuestiones externas y objetivas, al contrario de la Historia oral de vida que se ocupa de impresiones y subjetividades (Sebe, I998a). 
La tercera variante, correspondiente a la Tradición oral, se interesa por el individuo como vehículo de transmisión de los relatos antiguos y no se ocupa de las vivencias del presente (Sebe, I998a).

En relación a la memoria, diferentes autores elaboraron sus propuestas teóricas. Vansina (1985) y Halbwachs (2004) se refieren a la Memoria colectiva; Le Goff (I99I) a la Memoria histórica y Bergson (2006) aborda la Memoria psicológica. En los últimos decenios se han sumado amplias discusiones sobre los conceptos de posmemoria y memoria cultural, tampoco se puede olvidar el concepto de Lugares de memoria de Pierre Nora (1992) ampliamente derivado de las propuestas de Halbwachs y del "momento memorable" -François Dosse (2003)- que actualmente resulta crucial en las relaciones entre historia y memoria.

La Memoria colectiva, según plantea Jan Vansina (1985), es la más cercana a la historia oral, pues en ambas el origen del conocimiento de la realidad del grupo son las raíces míticas de la explicación del mundo. Por su parte, Maurice Halbwachs (2004) la relaciona con los recuerdos o ideales que componen la identidad comunitaria de un grupo y que son preservados por él. Estos trazos de la memoria son subjetivos, comprometidos con el pasado común y existen independientemente de los documentos escritos, monumentos u otros objetos materiales.

Vansina (1985) considera que algo muy importante en los trabajos de tradición oral es la convivencia con el grupo y la observación, pues gracias a ello se pueden enriquecer los testimonios como base del trabajo. El autor propone el concepto de los outsiders como investigadores e insiders, creados y entrenados en el propio grupo. La referencia directa a esto es la propuesta de etic/emic recogida de la psicología por Marvin Harris, que después recuperó Clifford Geertz (Schaffhauser, 20I0). Menciona también que la tradición oral y la memoria colectiva son diferentes en la manera como captan los relatos y en la forma que el historiador se vincula con el grupo seleccionado.

La memoria colectiva se preocupa más por la comunidad y por la manera en cómo percibe la vida, por lo que la tradición oral tiene interés por los temas. Sebe (I998b) señala que: 
En el caso de la tradición oral se habla del estudio de las fundamentaciones éticas de grupos amenazados. En este sentido, pienso en la responsabilidad de los intelectuales de actuar en favor del derecho de voz de aquellos que hasta historiográficamente son silenciados, de aquellos que, para expresarse en el contexto general, necesitan de otros para promover su existencia en cuanto participantes del proceso histórico. En la Memoria colectiva, en cambio, son autores "de adentro" los que actúan en el sentido de presentar soluciones de sobrevivencia para el propio grupo. Estamos hablando, por lo tanto, de voces distintas: en la tradición oral los narradores se valen de la ayuda de "otros", en la Memoria colectiva las voces son las de los protagonistas (p. II).

Le Goff (I99I) explica que la Memoria histórica es una construcción artificial realizada por los especialistas del pasado. Es una producción basada en el conocimiento emanado de la documentación propuesta por terceros, que los historiadores están seleccionando actualmente para dejarnos ver el pasado. Los documentos de trabajo se dividen en convencionales y no convencionales, siendo los primeros aquellos que se encuentran en los archivos y en su mayoría impresos; mientras que los no convencionales son producto de la cultura material: monumentos, fotografías, artefactos, etc.

La Memoria psicológica, como la comprende Bergson (2006), se basa en el recuerdo y proceso de olvido. Para los psicólogos bergsonianos la memoria es un continuo del cual es posible registrar solo fragmentos que se coleccionan en la memoria y construyen un material que los psicólogos tratan de reunir y evaluar. Por lo tanto, la memoria psicológica sería un proceso de rememoración (Sebe, I998a).

Existen otras clasificaciones relacionadas con la historia oral, donde se ubican las ramas de acuerdo a la utilidad que tienen para el historiador. La Historia oral pura se ocupa solamente de los testimonios y se centra en lo que se ha expresado en ellos. La Historia oral híbrida trata de recolectar testimonios en combinación con otras fuentes. Existen muchos historiadores que no quieren aceptar la Historia oral pura, puesto que la consideran poco critica (Sebe, 1998a). 
Con relación al trato que se debería dar a las fuentes orales, se considera que debe ser igual que en las escritas. En ambas tenemos que admitir la subjetividad implícita en ellas y es necesario establecer su veracidad a partir de la consulta de todas las fuentes de información al alcance de los historiadores (Mariezkurrena, 2008).

En la historia oral la entrevista es uno de los métodos más frecuentemente utilizados por los historiadores, con la cual el investigador busca la información para su trabajo y el entrevistado asume la idea que su historia y puntos de vista se harán públicos. Se crea una situación artificial y la conversación no es espontánea.

Existen varios requisitos para llevar a cabo una buena entrevista, como es el conocimiento previo del tema, la definición de la hipótesis y problemática de investigación, adecuada selección de informantes, actitud abierta para considerar nuevas vertientes de estudio y capacidad para llevar registro de los silencios y espacios vacíos. La estructura de la entrevista puede ser individual o grupal, y su estructuración en torno a un cuestionario fijo o flexible (Mariezkurrena, 2008).

El entrevistador elije el modelo de entrevista, los temas, la cantidad de informantes y el número de encuentros. El objetivo de una entrevista de historia oral es entender una vivencia, más que obtener datos. Las exageraciones, ficciones o fallas de la memoria del entrevistado aportan información importante acerca de la historia de su vida. El testimonio nos ayuda a comprender las vivencias íntimas de esa persona y posteriormente se debe saber interpretar adecuadamente (Mariezkurrena, 2008).

La elaboración del guion con una lista de ideas que se deben abordar en la entrevista es obligatoria. La importancia de los temas y el orden para abordarlos frecuentemente determina a la persona entrevistada y con base en los acontecimientos, se pueden plantear nuevas preguntas; por lo cual no es recomendable el uso de cuestionarios cerrados. Las expresiones corporales y los silencios aportan información adicional al 
testimonio hablado y en ellos debe fijar la atención el historiador oral (Mariezkurrena, 2008).

La duración de la entrevista es hasta que el investigador considera que la información recopilada lo satisface y cumplió con sus expectativas, pues un testimonio nunca se agota. Posteriormente iniciará con el análisis e interpretación, a partir de la transcripción de las grabaciones. Este proceso debe realizarse inmediatamente, de manera completa y fiel, pues la cercanía en el tiempo permite recuperar las palabras pérdidas o mal grabadas y registrar las impresiones acerca del informante, como son disposición, lenguaje corporal, etcétera (Mariezkurrena, 2008).

\section{Consideraciones metodológicas}

Para realizar el trabajo de investigación se tomó la decisión de hacer énfasis en el guion de entrevista y en el trabajo de campo, a partir de la lectura de la propuesta metodológica de Jan Vansina y Fritz Schütze (1983). Para atender al enfoque del primer autor, se modificó la narrativa biográfica hacia una narrativa cercana a la biografía, que permitió establecer al testigo en referencia cronológica de los hechos referidos. Además, pareció importante reforzar la observación con la fotografía.

En la investigación se dio importancia a la observación, como propone Vansina (1985), y en términos teóricos y metodológicos se usaron las estrategias que contribuyen en el desarrollo de una entrevista como narración, desde la perspectiva biográfica (Schütze, I983), tomando en cuenta las potencialidades y limitaciones que presentan.

Schütze (1983) considera que, gracias al uso de una teoría narrativa, se pueden elaborar los principios básicos e intersubjetivos que ayudan a estructurar una entrevista y que provocan el ordenamiento secuencial de los datos biográficos, que posteriormente pueden ser analizados. En una narración autobiográfica improvisada, el informante tiene confianza para abordar diferentes aspectos de su vida, lo cual es 
esencial para la entrevista. Se supone que este tipo de narración de experiencias personales sin una guía previa, permite a la persona acercarse lo más posible a los hechos reales (Muñiz, Roberti, Ambort, Bidauri, Riva y Viña, 20I5).

Para el propósito de este trabajo se prefirió denominar como entrevistas biográficas las entrevistas en profundidad semi-estructuradas, realizadas en Cuba. A partir de los relatos de los informantes se intentó recuperar la dimensión de diferentes eventos. El rol del entrevistador fue establecer un diálogo abierto para incitar al entrevistado a proporcionar respuestas cronológicamente precisas y claras. Fue necesario insistir en las referencias a terceras personas, ambientes y lugares en donde transcurren los acontecimientos biográficos (Pujadas, I992).

Riemann y Schütze (I992) consideran que dentro de la propuesta metodológica de la entrevista biográfica debe tomarse en cuenta el análisis y construcción de una teoría fundada, a partir de los relatos autobiográficos. Es importante crear condiciones de confianza y respeto entre el investigador y el informante para establecer un buen diálogo; explicar los fines para las cuales se realiza, resaltar la importancia de proveer la información y -finalmente- aclarar los aspectos en que estamos interesados.

Metodológicamente es importante analizar la veracidad de los acontecimientos contados, especialmente cuando se trata de eventos relacionados con personas reconocidas o significativas públicamente. Este procedimiento consiste en la revisión de la información recabada en las entrevistas, contrastándola con la de otras personas relacionadas con el tema y/o complementándola con fuentes escritas disponibles en los archivos. En la investigación realizada en Cuba esto no fue posible, simplemente por la falta de material escrito que abordara lo que a la vista parecen temas incomodas para el gobierno.

El procedimiento de análisis propuesto por Schütze (en Muñiz et al., 20I5) puede dividirse en los siguientes pasos: 
I. El análisis de los distintos tipos de texto; narrativos (cadenas de acontecimientos), descriptivos (situaciones, contextos) y argumentativos (explicaciones, razones);

2. El análisis formal; donde se identifican los sedimentos de las distintas experiencias biográficas, las secuencias temporales reconstruidas por el o la entrevistada;

3. La descripción estructural del contenido de los segmentos narrativos;

4. La abstracción analítica; la reconstrucción de la biografía como un todo, teniendo en cuenta los procesos estructurales de la historia de vida, las teorías biográficas propias del narrador o narradora y la relación de la biografía con el contexto global;

5. La comparación contrastada; comparación entre distintos estudios de caso. Desarrollo de categorías elementales a través de comparaciones mínimas (entre casos muy similares) y máximas (entre casos con diferencias contrastantes); y

6. La construcción de un modelo teórico; a partir de la comparación contrastada y las categorías emergentes se construyen núcleos teóricos, apoyándose en las teorías ya existentes que sustentan la investigación en curso (pp. 3I-32).

Los primeros tres pasos corresponden a la fase narrativa dentro de la cual es necesario tomar en cuenta los marcadores lingüísticos, para establecer la cronología. A partir del punto cuatro inicia el proceso de análisis, especialmente el que se relaciona con el contexto global. La interpretación de los datos debe realizarse junto con el informante para poder identificar y aclarar las contradicciones y discrepancias. En ese momento se deben realizar comparación basadas en el contraste de los casos mencionados y -finalmente- el investigador debe empezar la construcción de un modelo teórico, para el cual es necesario conocer las teorías existentes que apoyan el trabajo de investigación (Muñiz, et al., 20I5).

\section{Trabajo de campo}

Para el desarrollo de las actividades de investigación de campo se estableció como propósito recuperar y comprender la existencia y la 
percepción de la propaganda socialista soviética en Cuba. Para ello se definieron múltiples líneas de trabajo relacionadas con la presencia de la propaganda en la educación y la cultura de la Isla.

Dentro del trabajo de campo hubo situaciones que dificultaron el análisis y la comprobación de la información proporcionada por los testigos directos, relacionados con el sistema político de Cuba, pues la mayoría criticaron duramente al socialismo cubano, confundiéndolo con sovietismo ${ }^{2}$. Estas personas pidieron anonimato, quizás por temor a represalias de parte del gobierno, por lo cual se optó por recoger datos básicos como edad, género, profesión y lugar de residencia. En caso de autorización se puso el nombre sin apellidos o -de lo contrario- se consigna como anónimo.

Por las características mencionadas, los infamantes se dividieron en tres grupos: I) Personas mayores de 60 años y que todavía recuerdan cómo era la situación en Cuba antes, durante y después de la Revolución; 2) Personas de edad media comprendida entre 40 y 60 años; y 3) Menores de 40 años. En todos los casos se diferencia a los habitantes de zonas rurales y urbanas.

Los entrevistados del primer grupo recuerdan bien la presencia soviética en la Isla, específicamente en los años setentas y ochentas, cuando hubo varios especialistas que vinieron de la entonces Unión Soviética a apoyar en los aspectos económicos del país, principalmente. Mencionaron programas culturales de cine y televisión, así como las presentaciones de artistas. Lo que más les impactaba en aquel momento no era el mensaje político, sino las diferencias culturales entre cubanos y rusos, como llamaban a los soviéticos. Señalaron que los visitantes eran melancólicos, nostálgicos o que tomaban mucho, probablemente por la opresión que sufrían por parte de su gobierno o por el clima. Recordaban con mucha precisión nombres de políticos soviéticos de aquellos tiempos, sus funciones e impresiones que provocaban. También evocaban nombres de intelectuales y artistas que visitaron Cuba o que llegaban a través de los programas culturales. Al cuestionar la influencia soviética en la educación, mencionaban los nombres de Lenin, Stalin, Khrushov, Brezniev u otros miembros sobresalientes del 
aparato de poder de la Unión Soviética, a los que era común revisar en las clases. Todos, con mucha nostalgia, recordaban tiempos de su juventud y la Unión Soviética tomaba un lugar muy especial dentro de su historia personal. Las críticas al gobierno cubano no han cambiado su auténtica admiración por Fidel Castro.

Muchos ciudadanos, sobre todo los de edad más avanzada, dijeron que era a Fidel a quien debían agradecer por la educación, el cuidado de la salud, la vivienda, el alimento y su bienestar general. Señalaban que Cuba antes de la Revolución estaba dominada por la mafia italiana proveniente de Estados Unidos y predominaba la desigualdad y el racismo. Subrayaron que últimamente -después del año 2000- la desigualdad económica está de nuevo presente en la sociedad cubana, pues quienes tienen parientes en Miami o son empleados del gobierno, viven mejor que un ciudadano promedio, tienen acceso a las tiendas de lujo donde se vende productos extranjeros en dólares y pueden viajar a México, Estados Unidos o Rusia, donde compran cosas que no se consiguen en la Isla y posteriormente las venden. Por lo tanto, observan una creciente diferencia económica entre los habitantes, que antes del año 2000 no se notaba.

En el mismo grupo I resaltan la caída del sistema educativo y de salud, pero como fenómeno de hace apenas unos cuantos años y lo relacionan con la sistemática huida de la población fuera de Cuba, donde participan especialmente los profesionistas que se van en busca de mejores oportunidades. Les causa especial impacto la idea del gobierno de poner televisiones en los salones de clase en lugar de maestros o que se contrate a jóvenes recientemente graduados de las preparatorias, para que funjan como maestros en nivel medio superior.

172 En la entrevista con Javier, hombre de más de 60 años, señala que:

Agradezco a la Revolución, muchísimo, al Comandante. Fui de una familia muy pobre, fuimos ocho hermanos, mis padres trabajaban en el campo, era un tiempo muy duro. En principio mi padre no creía en la Revolución, pero cuando le dieron una propiedad y consiguió su propia casa, poco a poco cambio de opinión. Nosotros pudimos 
terminar las escuelas, mis dos hermanos se fueron a La Habana y allá terminaron la Universidad. De mis hijos solo uno se quedó en el campo, dos son las maestras y otro trabaja en turismo. Doy gracias a Fidel por todo lo que ha hecho para el pueblo y lo extraño mucho, para mi vive, vive en mi corazón. El gobierno de hoy ya no es como antes, solo piensan como enriquecerse. Ya no tienen las mismas ganas de ayudar al pueblo. Cuba está cambiando, no quiero que sea como antes de la Revolución (Javier, comunicación personal, noviembre, 20I8).

Es notoria, después de analizar esta y varias entrevistas, la dependencia hacia el gobierno. Esta postura es muy característica en los países socialistas y fue descrita por Zinoviev (1986) como homo sovieticus ${ }^{3}$. Todo, según palabras de Javier, se debía al Estado y -más aún- a Fidel. De hecho, el Estado y Fidel eran lo mismo, pues la pasión por Fidel era omnipresente, así como la tristeza por su muerte. Se temía el cambio de situación, ya que durante décadas se acostumbraron a que todas sus necesidades básicas fueran cubiertas por el gobierno.

Del grupo de edad entre 40 y 60 años, el entrevistado más joven criticaba más y admiraba menos los logros de la Revolución; también se burlaba de lo que según él era soviético, considerando que era una de las principales causas por las que Cuba estaba tan afectada, especialmente en lo económico. Los entrevistados mencionaron que la mayoría de las construcciones en la Isla que se hicieron en los años setentas y ochentas fueron gracias al apoyo soviético y, al mismo tiempo, reflexionaban que fue la causa de lo que llaman aflojamiento del gobierno cubano en la inversión y desarrollo de la industria nacional. En uno de los diálogos se dijo que: "Se recibía todo de allá (Unión Soviética o países del Bloque de Europa del Este), por ello se acostumbraron a no hacer nada (el gobierno). Hay dos bloqueos uno de Trump y otro de gobierno cubano, así es más fácil controlar a la gente" (Anónimo I, comunicación personal, noviembre, 20I8). También mencionaba con sarcasmo que la mafia italiana, antes de la Revolución, hizo más construcciones en la Isla que el gobierno cubano en décadas. En el mismo diálogo señala: "Vez el túnel (en La Habana) lo hicieron italianos y el Monumento a José Martí también, solo que lo inauguró Fidel" (Anónimo I, comunicación personal, noviembre, 20I8). Les daba tristeza que La Habana Vieja esté tan descuidaba, por lo que culpan al 
gobierno actual, pero también recuerdan que en los años noventa, cuando hubo muchas manifestaciones contra el gobierno y muchas pruebas de escape, en una ocasión llegó Fidel en su Jeep, hasta donde estaban las personas descontentas reunidas en el malecón. Entró solo con su chofer y se impuso a la multitud, abriendo su camisa sin chaleco antibalas y diciendo a los presentes que disparan, con lo cual la gente se impresionó. El entrevistado reflexionaba que así era Fidel, único. Recordó las represalias de los años 90 del siglo pasado: "Arrestaron gente y los fusilaron, no sé cuántos, me parece que cinco, para intimidarlos, pero de todos modos la multitud se les iba a Miami, hasta en las llantas que tiraban por el malecón, quien sabe a cuántos se los comieron los tiburones" (Anónimo 2, comunicación personal, noviembre, 20I8).

El otro informante recordaba que "Sí hubo mucho sobre la Unión Soviética en los medios de comunicación; también venían los trabajadores y los artistas. No me gustó mucho, prefiero Tschaykovsi o Tolstoi que el arte soviético. Muchos se casaron con la gente de aquí y se quedaron, otros se fueron a vivir allá" (Anónimo 2, comunicación personal, noviembre, 20I8). "Muy toscos, nosotros aquí estamos acostumbrados a lo tropical, a los colores, al realismo mágico en el arte, al Alejo (Carpentier). Aquí hay mucha África, todo es vibrante. Sí los veía y escuchaba, pero no me marcaron" (Anónimo 3, comunicación personal, noviembre, 20I8).

Al preguntarles por la influencia en educación mencionaron los mismos nombres que el grupo anterior y todos coincidieron en que en los años setentas y ochentas las escuelas tenían mucho mayor nivel que ahora. También se declararon castristas; solo uno de ellos, la persona más joven, de 42 años, tenía dudas sobre su orientación política. El

174 sovietismo les parecía un lejano recuerdo de un mundo que se terminó y jamás regresaría. De hecho, mejor que el actual.

Caminando rumbo a la Universidad de La Habana se entrevistó a un trabajador que reparaba las instalaciones eléctricas. Después de varios días de plática empezó a tener mayor confianza y dijo respecto al sistema de salud: 
El Hospital Universitario está muy mal, mi hija que tiene I6 años, estaba en las prácticas en el interior de la Isla y allá junto con sus compañeros contrajeron dengue. Como se empeoró su estado, la trasladaron a La Habana y ahorita está aquí. Ni siquiera tienen medicamentos para los tratamientos, ni pastillas contra el dolor. Yo gano doce dólares al mes y de esto tengo que mantener a mi familia, comprar comida y medicinas para mi hija que está hospitalizada. ¿Ya vio cuánto cuestan las pastillas? Así somos muchos que tenemos enfermos y sin la posición privilegiada. Muy mal esta todo esto. Antes no era así, algo hacían por el pueblo, pero cuando se envejeció y enfermó Fidel, Raúl tomó el poder en sus manos. Los campesinos le decían "La China" en tiempos de la Sierra Maestra, porque le gustaban los jóvenes, ¿no sabía? Por eso sucedió el conflicto entre Fidel y Raúl y por eso ahorita promueven matrimonios entre homosexuales, también para ganar más dinero de los extranjeros. Su hija (de Raúl) se declaró lesbiana o bisexual y ahorita lucha por sus derechos. Esto es lo que les interesa, hacer negocios con todo, no cuidar al pueblo. Venga conmigo a visitar a mi hija para que vea que no estoy mintiendo (Anónimo 4, comunicación personal, noviembre, 2018).

De hecho, se aprovechó la oportunidad y se visitó el hospital, que en efecto estaba como decía el hombre. Fue una de las pocas ocasiones en que se pudo comprobar la veracidad del relato de los entrevistados, tomando en cuenta la falta de fuentes oficiales y la censura para comentar las fallas estructurales del sistema político cubano.

En una oportunidad que hubo durante un viaje que se hizo al interior de Cuba, se encontró a Antonio, campesino de 54 años, quien gozaba de muy buena posición económica, gracias a la ayuda de sus hijos que escaparon a Miami. Dada los escases alimentarios y las facilidades del gobierno a favor de la apertura hacia la iniciativa privada, apostó por el cultivo de tomates a gran escala. En su relato menciona:

Mi papa era cafetalero, antes de la Revolución, aquí se da muy buen café. No fuimos ricos, pero nos fue bien. En 1959 quiso escapar, pero mi mamá estaba embarazada de mi hermano y presentaba alto riesgo de perder el producto, por eso no nos fuimos. Le quitaron mucha tierra, una parte la repartieron a la gente que finalmente no la cultivó y ahorita me la rentan a mí y la segunda parte se quedó en manos de 
Estado. Allá también cultivaron café. Después ya no les convenía el café y quitaron los cafetales para sembrar caña. En su tiempo todo era monocultivo en Cuba, pero se cayó la Unión Soviética y dejaron de vender, porque todo se iba para allá. Los campesinos dejaron todo en el abandono. Hace apenas unos años que empezaron a permitir que se cultivara lo que uno quisiera, solo que lo compran ellos. Por esto comencé con tomates, pero otra vez no los quieran comprar y no puede venderlos por mi cuenta, solo una parte. El resto se pudre, ¿ive allá? son mis tomates. Y los tomates hacen falta en La Habana (Antonio, comunicación personal, noviembre, 2018).

De hecho, a finales de noviembre de 2018 hubo escases de verduras, entre ellas los tomates, y se dijo en las noticias de televisión que era a causa del bloqueo norteamericano.

En el último grupo de personas solamente se realizaron dos entrevistas, con personas de entre 24 y 35 años. No tenían ningún interés en recordar algo acerca del sovietismo, pues todo lo pasado lo criticaban. Fidel Castro junto con Marx, Lenin o Stalin les parecían obsoletos. Ni siquiera soñaban con irse a Estados Unidos, su mente estaba enfocada en Europa. Por el malestar económico y político culparon al sovietismo, del cual sabían muy poco y tampoco tenían interés de conocerlo, pues criticaban la influencia que ejerció en Cuba. La más joven de las entrevistadas (24 años), al ser cuestionada sobre la influencia soviética en la educación, se río y respondió: “¿Cuál influencia?, yo ni siquiera tenía maestro, nos pusieron tele en la clase, ¿se imagina?, en lugar de la persona. Se les fueron casi todos en el periodo especial. Como maestros preparaban a egresados de la prepa y algunos de mis compañeros tenían como profesor a otro joven de 20 años. No me interesa ni el socialismo y menos el sovietismo. Fidel Castro fue un mito más, desgastado, de la historia cubana (Aurora, comunicación personal, 176 diciembre, 20I8).

Los jóvenes cuestionaban también la postura pasiva de la gente mayor, a quienes calificaban como faltos de voluntad propia, como mencionó uno de los estudiantes de la Facultad de Filosofía de la Universidad de La Habana: 
Cuando me titule me voy de aquí a Alemania. Muchos de mis conocidos consiguieron una novia europea y viven allá. Hay muchas turistas que vienen en busca de sexo y novio, uno tiene que aprovechar. Fidel se murió y algunos todavía lo adoran, ya es historia. Los veo haciendo cola por todo y esperando que el gobierno, como antes, les de lo que necesitan. Me va bien porque me regalan cosas mis novias y después las vendo. Lo que necesito se lo compro a mis amigos en el mercado negro. Y los viejos todavía hablando de Fidel y como era antes. Yo ni recuerdo aquellos tiempos, cuando nací, Fidel ya era viejo y todas las decisiones eran tomadas por él. De los ministerios se apoderaron gente de Raúl y todos los militares haciendo sus negocios (Pablo, comunicación personal, diciembre, 2018).

Conforme transcurría el tiempo de la entrevista, muchos cubanos se abrían más para comentar aspectos hasta cierto punto delicados. Se quejaban de la falta de alimento, ropa, internet y teléfonos celulares; de la corrupción de los funcionarios; y pocas veces de la falta de democracia, que parecía algo sin importancia, comparando con los problemas diarios que les aquejaban. Las entrevistas fueron para los cubanos una especie de catarsis que les permitiría seguir adelante. Se aprovechó de esta oportunidad para conocer cómo percibían el presente que les permite hablar del pasado, y cómo el presente está influido por el pasado; pues como observó Jan Vansina (1985) el presente es solo un aliento de pasado.

Una y otra vez dentro de las entrevistas se escuchaba el dicho: Pueblo chico, infierno grande. Muchos manifestaban que ya no les importaba tanto que pudieran ser vigilados por el gobierno, bromeando que con las carencias en Cuba seguro la policía ni tiene para compra de micrófonos o para los espías. Las entrevistas, que en la mayoría eran anónimas, permitieron hablar con libertad y sarcasmo acerca de la vida en la Isla, desafiando varias veces a la autoridad, emitiendo opiniones totalmente contrarias a las posturas oficiales. Al profundizar en el diálogo, abordaban temas prohibidos, especialmente los relacionados con la creciente desigualdad social. Subrayaban que uno de los logros más grandes de la Revolución fue la eliminación de las desigualdades y el racismo, que a lo largo de casi tres décadas originaron una de las sociedades más igualitarias del mundo. Al mismo tiempo reflexionaban 
también que, junto con la descomposición del Bloque del Este y el fin de la ayuda económica que recibían, se presentaron los problemas de sobrevivencia que con el tiempo empezaron a producir falta de equilibrio social. La corrupción escondida y el nepotismo dentro del gobierno se hicieron cada vez más visibles.

Divari, joven de 28 años entrevistado a finales de noviembre de 20I8, habló sin temor acerca del acaparamiento de la familia Castro de los mejores negocios en Cuba.

Sí, hay apertura económica, pero controlada por el Estado. Los mejores negocios se quedan en sus manos. Son accionistas de los hoteles de cadenas extranjeras, de los restaurantes y panaderías en los lugares más concurridos de la ciudad. Casi todos los altos puestos de gobierno se repartieron entre familiares, amigos y aliados. El nuevo presidente es títere de Raúl. Y los que no tienen conocidos bien posicionados, felices de recibir migajas. Cada vez más se nota que unos tienen y otros no, todavía unos años atrás no era tan notorio. Pero mis papás y muchos otros de su generación creen en el mito del gran Fidel y culpan al gobierno actual. No quieren ver que todo se empezó a podrir mucho antes. Mira los condominios, los construyeron soviéticos en los años setentas y desde entonces nadie les dio mantenimiento, no es algo nuevo la corrupción (Divari, comunicación personal, noviembre, 20I8).

Varios entrevistados hablaban acerca de la injusticia y desigualdad, fenómenos que se hicieron notar cada vez más, después del periodo especial. ${ }^{4}$ Para poder resistir, el gobierno tomó la decisión de reformar el sistema económico y poco a poco abrirlo para la inversión privada extranjera. La gente empezó a encontrar trabajos mejor pagados que en las empresas estatales y daban la posibilidad de entrar en el mercado negro. Apareció también otra oportunidad en el turismo sexual. Los que supieron moverse o que tenían conocidos influyentes, comenzaron a salir adelante. Era notorio que se vestían y comían mejor, que remodelaban sus casas.

La gran promesa y logro del Estado, que fue la educación, tampoco garantizaba un trabajo bien pagado. La desilusión del proyecto 
revolucionario cubano se notaba casi en todas las entrevistas, pues señalaban que se trataba de sobrevivir o huir.

A todos los entrevistados les molestaba la creciente desigualdad y se preguntaban a sí mismos por qué sucedía si fue contra lo que luchó la Revolución, qué era lo que había fallado. A pesar de pedir anonimato, los cubanos hablaron con sinceridad y critica, abordando más temas de los que se tenían previstos al principio de la entrevista. El problema más grande fue contrastar lo escuchado con fuentes escritas oficiales, pues los archivos visitados dentro de la estancia en la Universidad de La Habana, no comprobaban las versiones orales. Aquí se constató que el Estado ejerce control absoluto sobre la construcción de la historia oficial.

Otro aspecto de la excepcionalidad cubana es que los relatos, sobre todo los de gente de mayor edad, enfatizan tanto los placeres como las molestias de vivir en una sociedad comunista. Como ya reflexionaron en su publicación Pérez, Hernández y Trujillo (2018): "La estoicidad y el orgullo de la población han sido el factor y la cohesión social ante las condiciones adversas, que están presentes desde el inicio de la década de los años sesentas a la fecha" (p.4I).

\section{Conclusiones}

Como balance general de las narrativas obtenidas en las entrevistas y con base en las observaciones, se concluye que la presencia soviética en Cuba fue recordada por sus habitantes principalmente como una ayuda económica y no como influencia ideológica. No marcó de manera profunda la cultura y el estilo de vida cubano. Se destacó muy poco interés por el pasado en el caso de los jóvenes. Los más grandes de edad, con mayor preparación y habitantes de la ciudad, tenían más información y conocimiento acerca de la Unión Soviética. Los habitantes de las zonas rurales se enfocaban principalmente en la presencia física de los rusos en la Isla. Nadie se identificó con las ideas soviéticas dentro de la educación, excepto los especialistas en 
educación que reconocían la influencia soviética pedagógica. Para todos fue un periodo ya cerrado.

Las entrevistas fueron para las y los cubanos un pretexto para desahogar sus penas y al mismo tiempo evidenciaron que el control del Estado no es absoluto, pues el temor de las consecuencias por criticar y mantener una postura diferente a la oficial, no influyó mucho en la información proporcionada. En este punto, hubo una diferencia notable en la historia oral con respecto a los países detrás de la Cortina del Hierro, donde este campo de la historia no se pudo desarrollar ampliamente, precisamente por el miedo de la población a las represalias.

\section{Referencias}

Anderson, P., \& Blackledge, P. (2004). Marxism, and the New Left. Merlin Press.

Anónimo I. (20I8, noviembre). Entrevista realizada por Izabela Tkocz. La Habana, Cuba.

Anónimo 2. (2018, noviembre). Entrevista realizada por Izabela Tkocz. La Habana, Cuba.

Anónimo 3. (2018, noviembre). Entrevista realizada por Izabela Tkocz. La Habana, Cuba.

Anónimo 4. (2018, noviembre). Entrevista realizada por Izabela Tkocz. La Habana, Cuba.

Antonio. (2018, noviembre). Entrevista realizada por Izabela Tkocz. Matanzas, Cuba.

Aurora. (20I8, diciembre). Entrevista realizada por Izabela Tkocz. La Habana, Cuba.

Avilés, J. (20I6), El fin de "homo sovieticus." Recuperado de: https://elcultural.com/El-fin-del-homo-sovieticus.

Bergson H. (2006). Materia y memoria. Ensayo sobre la relación del cuerpo con el espíritu. Editorial Cactus. 
Bertaux D., Rotkirch A. y Thompson P. (2004). On Living Through Soviet Russia, Routledge. En L. Passerini, Memory and Totalitarianism. New Brunswick-Londres.

Dabrowski, R. (2008). Historia mowiona. Narodowe Centrum Kultury.

Divari. (20I8, noviembre). Entrevista realizada por Izabela Tkocz. La Habana, Cuba.

Dore E. (20I2). Historia oral y vida cotidiana en Cuba, Nueva Sociedad, Nuso No 242, 2012, Recuperado de:

https://nuso.org/articulo/historia-oral-y-vida-cotidiana-encuba/

Dosse F. (2003). La historia: Conceptos y escrituras. Buenos Aires:

Ediciones Nueva Visión.

Gusdorf, G. (1957). La palabra. Galatea: Nueva Visión.

Halbwachs, M. (2004). La memoria colectiva. España: Prensas.

Hebdige, D. (1979). Subculture: The Meaning of Styl. Routledge, Inglaterra.

Javier. (20I8, noviembre). Entrevista realizada por Izabela Tkocz. Matanzas, Cuba.

Le Goff, G. (I99I). El orden de la memoria; el tiempo como imaginario. España: Paidós Ibérica.

Lewis, O. (1980). Viviendo la revolución. México: Joaquín Mortiz.

Lewis, O., Lewis, R., \& Rigdon, S. (1977a). Four Men: Living the Revolution: An Oral History of Contemporary Cuba. University of Illinois Press.

Lewis, O., Lewis, R., \& Rigdon, S. (1977b). Four Women: Living the Revolution: An Oral History of Contemporary Cuba [Living the Revolution, v. 2]. University of Illinois Press.

Mariezkurrena Iturmendi, D. (2008). La historia oral como método de la investigación histórica. Geronimo de Uztariz, (23-24), 227-233.

Muñiz Terra, L., Roberti, E., Ambort, M. E., Bidauri, M. P., Riva, F. y

Viña, S. (2015). De la entrevista guionada a la entrevista biográfico-narrativa: reflexiones en torno a un trabajo de campo colectivo. En IV Jornadas Internas CIMeCS. La Plata.

Nora, P. (1992). Los lugares de memoria. Uruguay: Ediciones Trilce. 
Pablo. (20I8, diciembre). Entrevista realizada por Izabela Tkocz. La Habana, Cuba.

Pérez, F., Hernández, G., y Trujillo, J. (2018). Cuba en movimiento: derrumbe del socialismo, una mirada de primer orden. Revista Ratio Juris, I3(27), 27-44.

Perks, R., \& Thomson, A. (2006). The Oral History Reader. New York: Routledge.

Piovani, J. (2007). El diseño de investigación. En Marradi, Archenti y Piovani, Metodología de las ciencias sociales. Buenos Aires: Emece.

Pujadas Muñoz, J. J. (1992). El método biográfico: El uso de las historias de vida en ciencias sociales. México: Centro de Investigaciones Sociológicas.

Riemann, G., \& Schütze, F. (1992). Trajectory as a Basic Theoretical Concept for Analyzing Suffering and Disorderly Social Processes. In R. Maines, (ed.), Social organization and social process: essays in honor of Anselm Strauss (333-357). New York: Gruyter. Recovered from: https://nbnresolving.org/urn:nbn:de:0I68-ssoar-72I4.

Schaffhauser P. (2010). Aurora González Echevarría, La dicotomía emic/etic. Historia de una confusión. Relaciones. Estudios de historia y sociedad, 3I(I2I), p. 257-269.

Sebe Bom Meihy, J. C. (I998a). Manual de historia oral (ed. 2). Sao Paulo: Ed. Loyola.

Sebe Bom Meihy, J. C. (I998b). Definiendo la historia oral. Recuperado de: https://estudioshistoricos.inah.gob.mx/revistaHistorias/wpcontent/uploads/historias_30_8-I3.pdf

Schütze, F., (1983). Biographieforschung und narratives Interview. Neue Praxis, (3), pp. 283-294.

182 Thompson, P. (1978). The voice of the past. Oxford: University Press. Topolski, J. (1983). Teoría del conocimiento histórico. Polonia: PWN. Vansina, J. (1985). Oral Tradition as History. Madison: University of Wisconsin Press.

Zinoviev, A. (1986). Homo sovieticus. Grove/Atlantic. 


\section{Notas:}

\section{${ }^{\mathrm{I}}$ Se entiende como régimen postcastrista al gobierno cubano conformado después de la muerte de Fidel Castro.}

${ }^{2}$ El sovietismo es el nombre que se le da al sistema económico generalizado en el escindido Bloque del Este, tras la Segunda Guerra Mundial. Con el apoyo a los movimientos nacionalistas del llamado Tercer Mundo, intentó establecer una competencia con el capitalismo durante las décadas de I960 y principios de I970.

3 "El Homo sovieticus, un término sarcástico difundido a partir de los años 70 para aludir al peculiar tipo humano generado por la implacable y prolongada tiranía soviética, se vio enfrentado, a partir de las reformas de Gorbachov y sobre todo de la disolución de la Unión Soviética, a un entorno drásticamente nuevo, el de la libertad, al que no parece que le haya resultado fácil adaptarse" (Avilés, 20I6, párr. 3).

${ }^{4}$ Periodo entre 1990 y I994, después del derrumbe de la Unión Soviética, cuando abruptamente se cae la economía cubana.

Este artículo se publica bajo una licencia de Creative Commons Reconocimiento-NoComercial 4.0 Internacional, y puede ser usados gratuitamente para fines no comerciales, dando los créditos a los autores y a la revista.

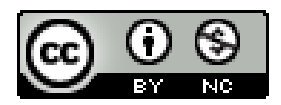

